\title{
Near-Edge X-Ray Absorption Fine Structure Spectroscopy of Agarose with a Compact Laser Plasma Soft X-Ray Source
}

\author{
T. FoK ${ }^{a, *}$, P. WAChulak ${ }^{a}$, K. JanuleWicz ${ }^{a}$, M. Duda $^{b}$, E. WęGrzyŃSKi $^{a}$, \\ A. BARTNIK ${ }^{a}$, R. JAROCKI ${ }^{a}$ AND H. FiedoroWICZ ${ }^{a}$ \\ ${ }^{a}$ Institute of Optoelectronics, Military University of Technology, S. Kaliskiego 2, 00-908 Warsaw 49, Poland \\ ${ }^{b}$ Faculty of Nuclear Sciences and Physical Engineering, Czech Technical University in Prague, \\ Břehová 78/7, 11519 Prague, Czech Republic
}

\begin{abstract}
In this work, we demonstrate near-edge X-ray absorption fine structure spectroscopy of agarose with a compact laboratory laser-plasma soft X-ray source. The source is based on a double stream gas puff target irradiated by nanosecond laser pulses. The emission spectra of the source and the absorption spectra of the investigated sample were measured simultaneously using a flat-field spectrometer. Soft X-ray emission from a krypton/helium target in the range between 1.5 and $5 \mathrm{~nm}$ was applied as the source. The transmission measurements revealed the specific spectral features near the carbon $K-\alpha$ absorption edge corresponding well to the results obtained with synchrotron radiation.
\end{abstract}

DOI: 10.12693/APhysPolA.137.51

PACS/topics: soft x-rays, spectroscopy, absorption

\section{Introduction}

Near-edge X-ray absorption fine structure spectroscopy (NEXAFS) is a well-known and established method employed for compositional analysis. It is based on spectral absorption measurements spanning the energy range from a few eV below the absorption edge of the studied element to typically a few tens of $\mathrm{eV}$ above the edge in the soft X-ray spectral region [1]. This is achieved by probing the electronic transition from core levels to unoccupied states. Each element has a characteristic energy of core bonding, therefore NEXAFS spectra contain element-specific information. Additionally, the energy levels of initial and final states are strongly dependent on the involved molecular bonds [2]. Currently, the majority of large-scale facilities have a beamline dedicated to studying X-ray absorption. Synchrotron light has allowed us to apply NEXAFS methods to various fields of science, studying both organic [3], inorganic [4], and nanoscale [5] samples. Also, laser-produced plasma (LPP) or higher order harmonic generation (HHG) light sources based on solid [6] or single gas jet [7] were employed for this type of experiments.

In this work, we demonstrate the possibility to perform table-top NEXAFS measurements on a thin organic sample (agarose) with a laser-plasma SXR source, based on a double stream gas puff target, emitting SXR radiation from a krypton/helium plasma. Two exposure conditions are compared: a few tens of seconds of exposure

*corresponding author; e-mail: tomasz.fok@wat.edu.pl time (200 SXR pulses) with a laser pulse energy below $1 \mathrm{~J}$ is compared with a single shot (SXR pulse) regime with a laser pulse energy of a few joules. The experimental data compare well with simulations performed using the fdmnes [8] software and are in agreement with synchrotron data.

\section{Experimental setup}

The general experimental setup for the SXR NEXAFS measurements of agarose using the $\mathrm{Kr} / \mathrm{He}$ plasma emission is depicted in Fig. 1. The experiment was performed with two different pump lasers, used to create laser plasma. The first (small) laser was an Nd:YAG laser, model NL 303 HT (EKSPLA, Lithuania), which delivers pulses with an energy of $0.65 \mathrm{~J}$ and $3 \mathrm{~ns}$ pulse duration that are focused by a lens with $f=2.5 \mathrm{~cm}$ focal length onto a $\mathrm{Kr} / \mathrm{He}$ double stream gas puff target. The second (large) laser, model NL129 (also EKSPLA), delivers pulses with an energy up to $10 \mathrm{~J}$ and down to 1 ns pulse duration focused on the gas puff using a lens with $f=10 \mathrm{~cm}$ focal length. Both works with $10 \mathrm{~Hz}$ repetition rate but in the second case the NEXAFS measurement was performed in single shot regime.

A double stream gas puff target is formed by a collinear set of two nozzles, driven independently by two electromagnetic valves. The diameters of the nozzles are $0.4 \mathrm{~mm}$ for the inner nozzle and $0.7-1.5 \mathrm{~mm}$ for the outer, ringshaped nozzle. In the case of the small laser, the inner nozzle was pressurized with krypton gas at an optimum backing pressure of 3 bar, while the outer nozzle was connected to helium pressurized to 5.5 bars. The optimal pressures in case of the large laser were 11 bar and 5 bar, respectively. 


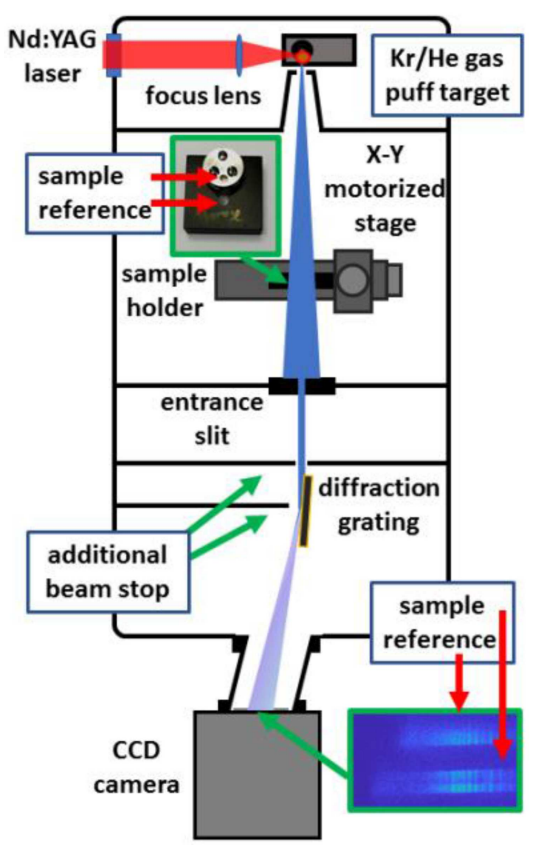

Fig. 1. The experimental NEXAFS measurements of agarose using a laser-plasma source based on a double stream gas puff target.

The radiation from the krypton plasma, without any filters, illuminates the sample under investigation. The sample holder (Fig. 1) allows for the SXR radiation to illuminate the sample to obtain absorption spectrum, but at the same time has small orifice, just below the sample, which allows the direct reference signal (spectrum) to be also recorded. Thus, in this design, two signals enter the spectrometer slit and are diffracted towards the CCD camera by the grazing incidence diffraction grating. The in-house developed SXR spectrometer was equipped with a $12 \mu \mathrm{m}$ slit, Hitachi (High Technologies America, Inc., USA) flat field diffraction grating (2400 lines per mm), and a back-illuminated CCD camera (GE 20482048, greateyes, Germany). During spectrometer calibration the resolving power of the SXR spectrometer $E / \Delta E$ was estimated to be $\approx 940[9]$.

\section{Agarose near-edge X-ray absorption spectroscopy results}

Agarose is a linear polysaccharide made up from alternating D-galactose and 3,6-anhydro-alpha-Lgalactopyranose residues, joined by alpha- $(1 \rightarrow 3)$ and beta- $(1 \rightarrow 4)$-linkages [10]. Agarose is widely used in the industry, biotechnology, and molecular biology [11]. The sample was prepared by dissolving an agarose powder (Agarose for DNA Electrophoresis research-grade, SERVA, Germany) in distilled water (1:5 by weight). A $2 \mu$ l droplet was deposited using a standard micropipette on top of a $\mathrm{Si}_{3} \mathrm{~N}_{4}$ membrane (75 nm thickness, $1.5 \times 1.5 \mathrm{~mm}^{2}$ window size) and dried in a nitrogen atmosphere.

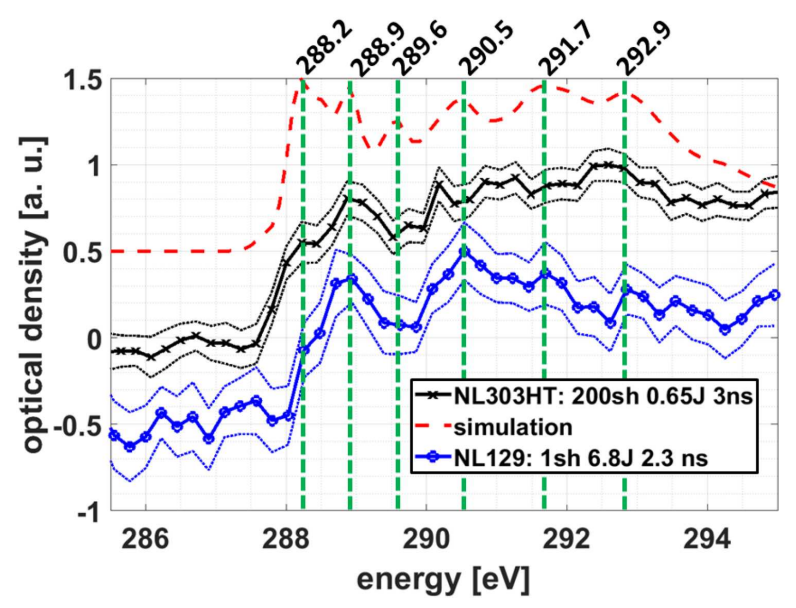

Fig. 2. Comparison of a normalized on differential absorption spectrum for agarose from simulation, 200 shots on NL303 HT and single shot at NL129.

The sample and reference spectra were acquired simultaneously during an exposure to $200 \mathrm{SXR}$ pulses at $10 \mathrm{~Hz}$ repetition rate (20 $\mathrm{s}$ long exposure) using the small laser and with a single $\sim 2$ ns SXR pulse using the large laser. The optical density extracted from the NEXAFS spectra of agarose recorded with the two available lasers are presented in Fig. 2. It shows the cases of: multiple pulse exposure (small laser, cross-mark spectrum) and single SXR pulse exposure (large laser, circular-mark spectrum). Results of simulations conducted applying the fdmnes procedure and based on crystallographic data available in [10] are given for comparison. The radius of the simulation was equal to $8 \AA$ and a Gaussian convolution function width was equal to $0.3 \mathrm{eV}$ (FWHM). In both simulation and the NEXAFS output it is possible to recognize highly correlated peaks at $288.2 \mathrm{eV}$ and $288.9 \mathrm{eV}$ $\left(1 s \rightarrow \pi^{*}\right)$ as well as 289.6, 290.5, 291.7, and $292.9 \mathrm{eV}$ $\left(1 s \rightarrow \sigma^{*}\right)$. The presence and proper energy calibration of the spectral features in both simulated and experimental data show a good agreement between simulation and experiment. Experimental agarose spectra are also in a qualitative agreement with the synchrotron data [12], although, the synchrotron-based measurements do not show all NEXAFS spectral features present in the simulations and in our experimental data. Most likely this is due to spatial localization of NEXAFS spectra acquisition and probably lower photon flux.

\section{Conclusions}

A proof-of-principle experiment, showing the applicability of a laser-plasma source based on a double-stream gas puff target for both multi- and single-shot NEXAFS was demonstrated, using agarose as sample. The obtained agarose spectra are comparable to simulated spectra and to those obtained with the synchrotron radiation. Simultaneous acquisition of both the sample and reference spectra allow highly accurate data collection 
and subsequent processing. Regardless of fluctuations in the source energy and mechanical instability of the system the processed data conserves the necessary features. Moreover, the demonstrated single-shot capability opens up the possibility of time-resolved NEXAFS studies on the nanosecond scale in the near future.

\section{Acknowledgments}

This work is supported by the National Science Centre, Opus programme, grant agreement number UMO-2015/17/B/ST7/03718 and UMO2015/19/B/ST3/00435 and from the European Union's Horizon 2020 research and innovation program, under Laserlab-Europe IV, grant agreement No. 654148. We also thank the Referees for their useful and constructive comments.

\section{References}

[1] V. Carravetta, O. Plashkevych, H. Ågren, J. Chem. Phys. 109, 1456 (1998).

[2] A. Gainar, J.S. Stevens, Ch. Jaye, D.A. Fischer, S.L.M. Schroeder, J. Phys. Chem. B 119, 14373 (2015).

[3] O. Plekan, V. Feyer, R. Richter, A. Moise, M. Coreno, K.C. Prince, I.L. Zaytseva, T.E. Moskovskaya, D.Yu. Soshnikov, A.B. Trofimov, J. Phys. Chem. A 116, 5653 (2012).
[4] I. Tanaka, T. Mizoguchi, T. Sekine, H. He, K. Kimoto, T. Kobayashi, S.D. Mo, W.Y. Ching, Appl. Phys. Lett. 78, 2134 (2001).

[5] T. Hemraj-Benny, S. Banerjee, S. Sambasivan, M. Balasubramanian, D.A. Fischer, G. Eres, A.A. Puretzky, D.B. Geohegan, D.H. Lowndes, W. Han, J.A. Misewich, S.S. Wong, Small 2, 26 (2006).

[6] P.J. Mallozzi, R.E. Schwerzel, H.M. Epstein, B.E. Campbell, Science 206, 4416, 353 (1979).

[7] F. Barkusky, A. Bayer, S. Döring, B. Flöter, P. Großmann, Ch. Peth, M. Reese, K. Mann, Proc. SPIE 7361, 736112 (2009).

[8] O. Bunau, Y. Joly, J. Phys. Condens. Matter 21, 345501 (2009).

[9] P. Wachulak, M. Duda, A. Bartnik, A. Sarzyński, Ł. Węgrzyński, M. Nowak, A. Jancarek, H. Fiedorowicz, Opt. Expr. 26, 8260 (2018).

[10] National Center for Biotechnology Information, PubChem Database, Sepharose, 2019.

[11] R. Meena, A.K. Siddhanta, K. Prasad, B.K. Ramavat, K. Eswaran, S. Thiruppathi, M. Ganesan, V.A. Mantri, P.V. Subba Rao, Carbohydr. Polym. 69, 179 (2007).

[12] S. Mitsunobu, M, Zhu, Y, Takeichi, T. Ohigashi, H. Suga, M. Jinno, H. Makita, M. Sakata, K. Ono, K. Mase, Y. Takahashi, Microbes Environm. 31, 63 (2016). 